\title{
Japanese Predicate Cleft Constructions as a Morphological Reduplication
}

\author{
Sachie Kotani \\ skotani@tezukayama-u.ac.jp \\ Tezukayama University / University of British Columbia
}

Japanese has predicate reduplication constructions (PRCs), which are also called Predicate Cleft Constructions (PCCs), as shown in (1), where two identical predicates appear.
(1) Ben-wa
(kinoo) jisyo-o
kau/kat-ta koto-WA
(*kinoo) kat-ta.
$\begin{array}{lll}\text { Ben-Top yesterday dictionary-Acc buy/buy-Pst Nml-CTop } & \text { (B) } \\ \text { 'Ben indeed bought a dictionary (yesterday). (But he returned it back.)' }\end{array}$

In this paper, I will observe how this kind of constructions behave and propose an analysis based on Morphological reduplication, comparing with other verb reduplication phenomena in Japanese.

PRCs have the following properties: (i) two identical predicates appear adjacent in the sentence final position, (ii) the first predicate must be followed by either one of the nominalizers $(\mathrm{Nml}),-k o t o,-n o$, and $-n i$, which is followed by the contrastive topic marker (CTop), $-W A$, and (iii) the second predicate must be inflected while the first one can in affirmative sentences. In addition, in negative sentences, two predicates must be completely identical and inflected, as in (2).

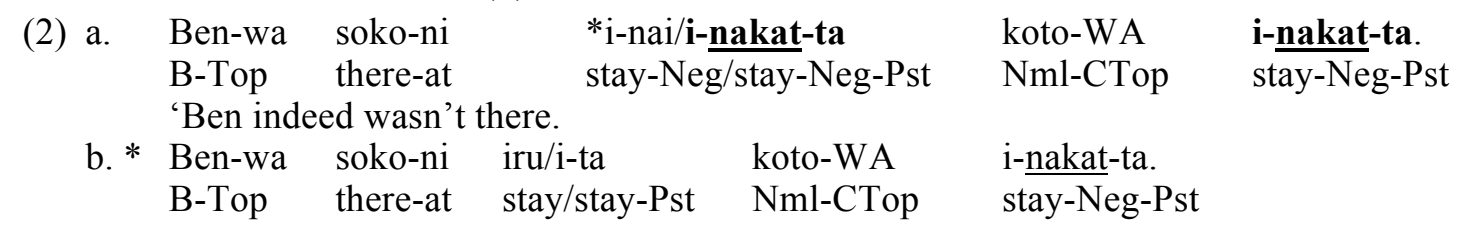

Furthermore, causative and potential morphemes prefer two predicates to be identical, and Passive morpheme requires two predicates to be identical, which is given in (3-5).
(3) a.
$\begin{array}{ll}\text { Ben-wa } & \text { Sean-ni } \\ \text { B-Top } & \text { S-Dat }\end{array}$
hon-o ?yomu/*yom-da
koto-wa
yom-ase-ta.
b. Ben-wa Sean-ni
book-Acc $\mathrm{read} / \mathrm{read}-\mathrm{Pst}$
Nml-CTop
read-Caus-Pst
B-Top S-Dat book-Acc read-Caus/read-Caus-Pst Nml-CTop
yom-ase-ta.
'Ben indeed made Sean read a book.'
(4) a. Ben-wa nihongo-o ?hanasu/*hanasi-ta koto-wa hanas-e-ta.

B-Top Japanese-Acc speak/speak-Pst Nml-CTop speak-Pot-Pst
b. Ben-wa nihongo-o *hanas-e-ru/hanas-e(r)-ta koto-wa
B-Top Japanese-Acc speak-Pot/speak-Pot-Pst Nml-CTop
hanas-e(r)-ta. speak-Pot-Pst
'Ben indeed could speak Japanese.'
(5) a. Ben-wa pasokon-o *nusumu/*nusum-da koto-WA nusum-are-ta. B-Top computer-Acc steal/steal-Pst Nml-CTop steal-Pass-Pst B-Top computer-Acc steal-Pass/steal-Pass-Pst Nml-CTop steal-Pass-Pst
b. Ben-wa pasokon-o *nusum-are-ru/nusum-are-ta koto-WA nusum-are-ta. 'Ben indeed had his computer stolen.' read-Caus-Pst 
(6) a. Ben-wa jisyo-o kau/kat-ta koto-WA jisyo-o kat-ta.

B-Top dictionary-Acc buy/buy-Pst Nml-CTop dictionary-Acc buy-Pst

'Ben indeed bought a dictionary. (But it was for kids.)'

b. Ben-wa hon-o kinoo kaesu/kaesi-ta koto-WA kinoo kaesi-ta.

B-Top book-Acc yesterday return/return-Pst Nml-CTop yesterday return-Pst

'Ben indeed returned a book yesterday. (But it was at midnight.)'

Importantly, PRCs do not allow the first predicate to appear in any other places, as in (7).

\begin{tabular}{|c|c|c|c|c|c|c|}
\hline (7) $\mathrm{a}$. & $\begin{array}{l}{ }^{*} \underline{[\mathbf{k a u} / \text { kat-ta }} \\
\text { buy/buy-Pst } \\
{ }^{*} \text { Ben-wa } \\
\text { Ben-Top }\end{array}$ & $\begin{array}{l}\text { koto-WA] } \\
\text { Nml-CTop } \\
\text { [kau/kat-ta } \\
\text { buy/buy-Pst }\end{array}$ & $\begin{array}{l}\text { Ben-wa } \\
\text { Ben-Top } \\
\text { koto-WA] } \\
\text { Nml-CTop }\end{array}$ & $\begin{array}{l}\text { jisyo-o } \\
\text { dictionary-Acc } \\
\text { jisyo-o } \\
\text { dictionary-Acc }\end{array}$ & $\mathrm{t}$ & $\begin{array}{l}\text { kat-ta. } \\
\text { buy-Pst } \\
\text { kat-ta. } \\
\text { buy-Pst }\end{array}$ \\
\hline
\end{tabular}

The observations above show that there is no evidence that tells syntactic movement derives the PRCs in Japanese, which seems to be different from PCCs observed in some other languages, such as Russian, Gbe languages or Hebrew (cf. Abels 2001, Aboh 2003 and Landau 2006).

It is well-known that Japanese has varieties of verb reduplications as shown below:

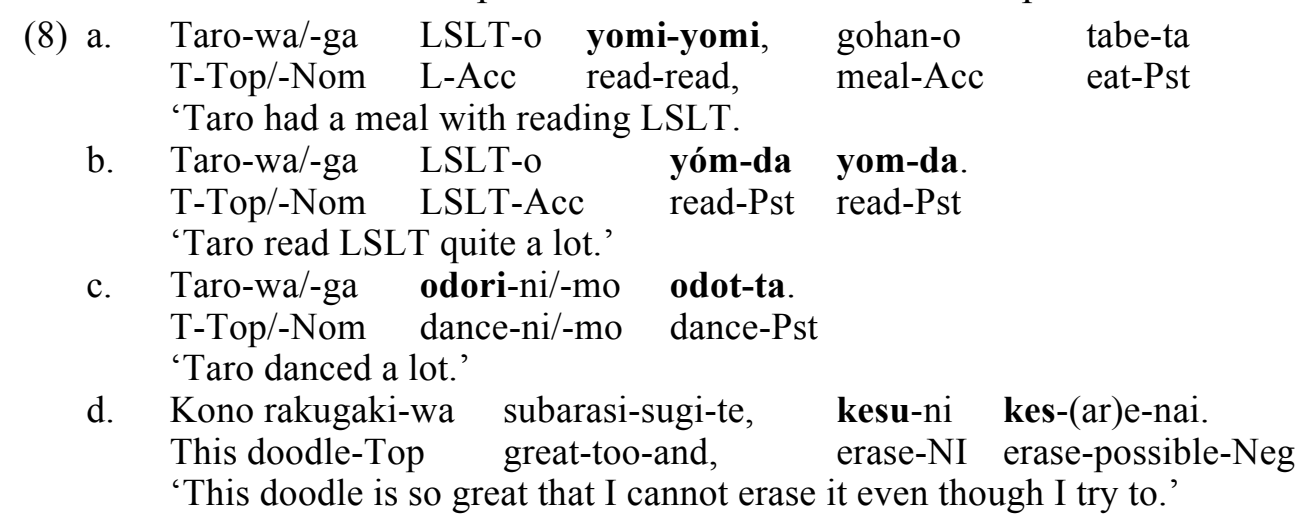

Comparing the productivity of these verb reduplications with that of Japanese PRCs, verb reduplications have restrictions on types of verbs to appear and requirements on the form or the number of morae of a verb, and they seem to have a certain meaning within the specific form. On the other hand, PRCs are completely productive with no restrictions on predicates at all.

I will argue below that there is only one predicate in the syntax, which is reduplicated after Spell-Out in the morpho-phonology domain. Predicate reduplication observed in PRCs is just a morphological effect to make the predicate marked by CTop.

Within the framework of Minimalist Program, I assume that Head-movement optionally applies in the syntax (cf. Chomsky 1995, Marantz 1997). Within the framework of Distributed Morphology, I also assume Morphological Mergers: (i) Lowering operates in terms of hierarchical structure, and (ii) Local Dislocation operates in terms of linear adjacency (Embick and Noyer 2001: 561). The CTop marker is assumed to be a focus sensitive particle adjoins to some phrase level.

Adopting Kotani (2009), I assume that there are two types of Head-movement in the 
syntax: Head-movement and Partial Head-movement, as in (9). With Head-movement, movement starts with the root, $\sqrt{ }$, up to $\mathrm{T}$ via $v$ successive cyclicly, as in (9a). With Partial Head-movement, movement starts with $v$ to $\mathrm{T}$, skipping the root, $\sqrt{ }$, as in (9b).

(9) a. Head-movement:

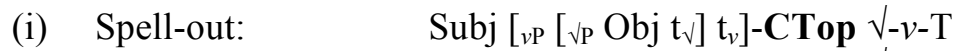

(ii) Morphology: $\quad \operatorname{Subj}\left[{ }_{v \mathrm{P}}\left[{ } \mathrm{P}\right.\right.$ Obj $\left.\left.\mathrm{t}_{\sqrt{ }}\right] \mathrm{t}_{v}\right]-$-CTop ${ }-v-\mathrm{T}$

b. Partial Head-movement:

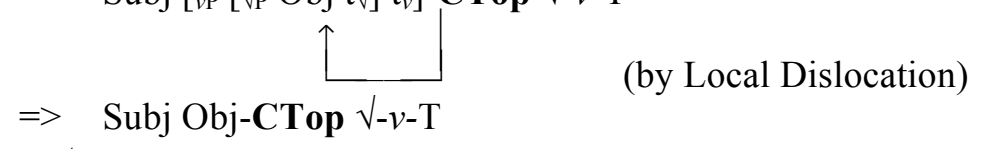

(i) Spell-out: $\quad$ Subj $\left[v_{\mathrm{P}}\left[{ }_{\mathrm{VP}} \mathrm{Obj} \downarrow\right] \mathrm{t}_{v}\right]$-CTop $v$-T

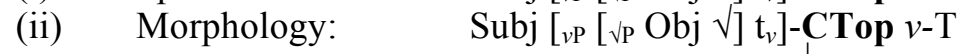

$$
\Rightarrow \quad \text { Subj Obj } \sqrt{ }-\text { CTop } v \text {-T }
$$

(by Local Dislocation)

(10a) is a result of Head-movement in (9a). (10b) is a result of Partial Head-movement in $(9 b)$.

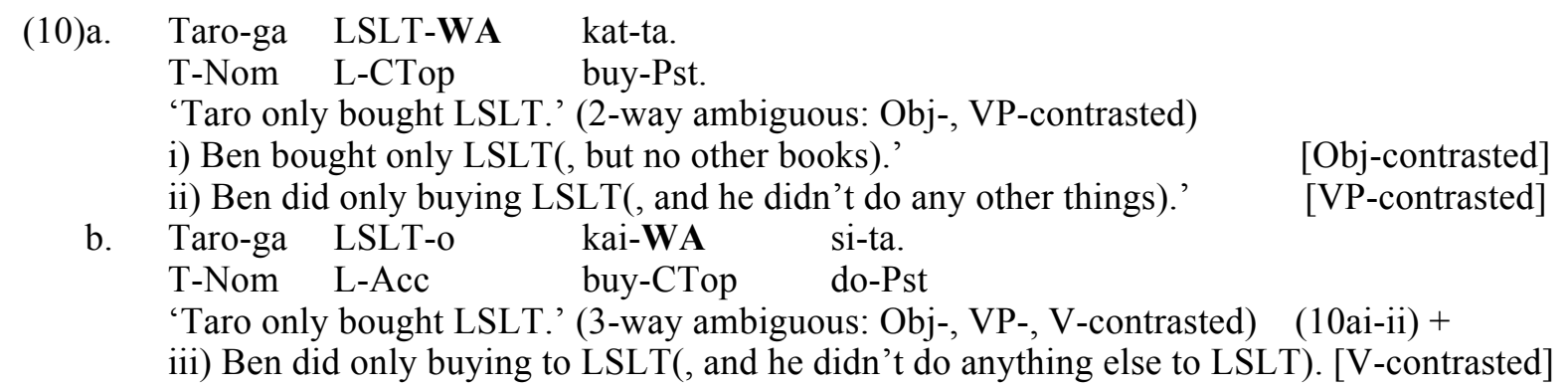

In the case of no head-movement applied in the syntax, the morphology assembles the heads and inserts a certain lexical item into the head-complex. When CTop adjoins $v \mathrm{P}$, we have $\mathrm{T}$ stranding with FP intervening between $v$ and $\mathrm{T}$ at Spell-out.

(11) No Head-movement when CTop adjoins $v \mathbf{P}$ :

(i) Spell-out: $\quad$ Subj $\left[{ }_{\nu P}\left[{ }_{v P} \mathrm{Obj} \downarrow\right] v\right]$-CTop T

(ii) Morphology: $\quad$ Subj $\left[v^{\circ}\left[v_{\mathrm{P}} \mathrm{Obj} \sqrt{ } v\right]\right.$-CTop T

$$
\Rightarrow \quad * \text { Subj Obj } \sqrt{ }-v \text {-CTop T }
$$

Note that CTop, $-W A$, only follows something non-verbal and comes at the end of a morpheme/word. This means that CTop cannot directly attach to $V_{-} v$ by itself because of the subcategorization mismatch. In addition, $\mathrm{T}$ cannot stand by itself. In order for the head-complex to be suffixed with the CTop marker $-W A$, the $n$ head must be inserted right before CTop.

(11') No Head-movement when CTop adjoins $v P$ :
(i) Spell-out:
(ii) Morphology:
Subj $\left[v_{\mathrm{P}}\left[{ }_{\mathrm{P}}\right.\right.$ Obj $\left.\left.\sqrt{ }\right] v\right]-$ CTop T
Subj $\left[v_{\mathrm{P}}\left[{ }_{\mathrm{P}}\right.\right.$ Obj $\left.\sqrt{ } \boldsymbol{v}\right]$-CTop T
$\Rightarrow \quad *$ Subj Obj $\sqrt{ }-v-\mathbf{C T o p} \mathrm{T}$
$\Rightarrow \quad$ Subj Obj [head-complex $\left.\underline{V_{-v}-n-C T O p} V_{-}-\mathrm{T}-\mathrm{T}\right]$

At the same time, $V_{-} v$ must be reduplicated to form $V_{-} v-T$ next to the nominalized one so that $\mathrm{T}$ can suffix to the stem to realize as a part of the head-complex and the predicate itself has no categorical change in the morphology even though the nominalizer is inserted. As a result, we have an example where the first and the second predicates are not identical like 
below:

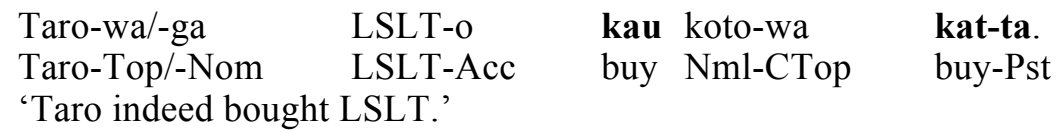

When CTop adjoins CP, we have the head-complex nominalized by a nominalizer inserted into C, which is followed by CTop at Spell-out.

\section{(13) No Head-movement when CTop adjoins CP:}

(i) Spell-out: $\quad$ Subj $\left[{ }_{v \mathrm{P}}\left[{ }_{\mathrm{vP}} \mathrm{Obj} \downarrow\right] v\right] \mathrm{T}$ C-CTop

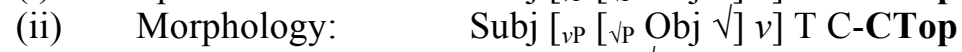

$$
\begin{aligned}
& \Rightarrow \quad * \text { Subj Obj } \sqrt{ }-\nu \text {-T-C-CTop } \\
& \Rightarrow \quad \text { Subj Obj [head-complex } \underline{V}-v \text {-T } n \text {-CTop } \sqrt{ }-v-T]
\end{aligned}
$$

Since CTop, $-W A$, cannot end a sentence, $\sqrt{ }-v-\mathrm{T}$ is reduplicated right after that. Such reduplication is inevitable in order to keep the head-complex [+V], with no category changed, in the morphology and in order for CTop to be suffixed to the head-complex. In this case, an example such as (14) is derived. The both predicates are identical in this example.

$\begin{array}{lllll}\text { Taro-wa/-ga } & \text { LSLT-o } & \text { kat-ta } & \text { koto-wa } & \text { kat-ta. } \\ \text { Taro-Top/-Nom } & \text { LSLT-Acc } & \text { buy-Pst } & \text { Nml-CTop } & \text { buy-Pst } \\ \text { 'Taro indeed bought LSLT.' } & & & \end{array}$

This analysis correctly predicts that nothing but a nominalizer followed by CTop can intervene between two predicates, as in (1), and that, if CTop associates with a predicate phrase including an argument/adjunct, the phrase should be reduplicated, as shown in (6). This analysis also explains the examples in (3-5) with no problem. Finally and most importantly, this analysis must predict that the V-contrasted meaning in (10b) and a PRC example in (1) must have the same meaning, which is correct.

\section{References}

Abels, K. 2001. The Predicate Cleft Construction in Russian. In Frank, S. et al. (eds.), Annual Workshop on Formakl Approaches to Slavic Linguistics: The Bloomington Meeting, 1-18. Michigan: Michigan Slavic Publications.

Aboh, E. O. 2003. The Morphosyntax of complement-head sequences. Clause Structure and word order patterns in Kwa. New York: Oxford University Press.

Chomsky, N. 1995. The Minimalist Program. Cambridge, MA: MIT Press.

Embick, D. and R. Noyer. 2001. Movement Operations after Syntax. Linguistic Inquiry 32, 555-595.

Kotani, S. 2009. Focus Particles and Their Effects in the Japanese Language. Ph.D. dissertation, University of Delaware.

Landau, I. 2006. Chain Resolution in Hebrew V(P)-fronting. Syntax 9, 32-66.

Marantz, A. 1997. No escape from syntax: Don't try morphological analysis in the privacy of your lexicon. UPenn Working Papers in Linguistics 4(2), 201-225. 